Note

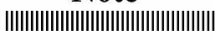

\title{
ESR Characterization of Irradiated Peony Root, A Medicinal Plant Containing Crystalline Sugars
}

\author{
Rumi YAMAOKI, Shojiro KIMURA and Masatoshi OHTA* \\ Osaka University of Pharmaceutical Sciences \\ 4-20-1 Nasahara, Takatsuki-shi, Osaka 569-1094, Japan \\ *Niigata University \\ 8050 Igarashi 2-no-cho, Nishi-ku, Nïgata-shi, Niigata Pref. 950-2181, Japan
}

Received October 15, 2012

\begin{abstract}
The ESR spectral property of irradiated peony root was studied during development of a system for detection of irradiated medicinal plants containing crystalline sugars. The plant had a weak ESR signal near $\mathrm{g}=$ 2.005 before electron beam-irradiation. After $10 \mathrm{kGy}$ irradiation, the line shape became broader and cellulose-like signal : a pair of lines at $6 \mathrm{mT}$ across the center signal near $\mathrm{g}=2.005$ and sugar-like spectrum : a broader line with more than $6 \mathrm{mT}$ of overall spectrum width, was observed. The ESR intensity decreased considerably at 30 days post-irradiation and was stable thereafter. The stable ESR line shape of irradiated peony root resembled that of irradiated sucrose, and the intensity 30 days post-irradiation increased linearly as a function of sucrose content. This result shows that the radical concentration correlation to sucrose content in irradiated plant material is predictable.
\end{abstract}

Key Words : ESR, irradiation, peony root, radiation-induced radical, sucrose

\section{Introduction}

Irradiation using $\gamma$-rays $\left({ }^{60} \mathrm{Co},{ }^{137} \mathrm{Cs}\right), \mathrm{X}$-rays less than $5 \mathrm{MeV}$ and electron beam (EB) less than $10 \mathrm{MeV}$, is a technique for reduction of microorganisms or disinfection of food or pharmaceuticals. It is a cold treatment without any substantial increase in product temperature and causes fewer changes in texture, color and flavor than heat treatment. By direct or indirect action of radiation, free radicals derived from the constituents, including water and lipids, are generated in the tissues. Most radicals quickly disappear by various recombination processes. However, a small proportion of radiation-induced radicals is trapped in the solid phase of substances, where they remain as stable radical components.
Electron spin resonance (ESR) is highly sensitive for detecting trace-level radicals in irradiated substances in a nondestructive quick assay. In the European Committee for Normalization, ESR analysis is approved as one of the standard reference methods for the detection of irradiated food, including those foods containing cellulose like pistachio nut shells, paprika powder and fresh strawberries, as well as foods containing crystalline sugar like dried figs, dried mangoes, dried papayas and dried raisins $^{1,2)}$. Cellulose-derived radical produces ESR signals : a pair of lines at $6 \mathrm{mT}$ across the central signal near $g=2.004-2.005$. Sugar-derived radicals, including mono- or disaccharides, produce broader ESR signal lines with more than $6 \mathrm{mT}$ of overall spectrum width (center of spectrum, near $g=2.004)$ and significant asym- 
metrically divided signal line shapes. In many irradiated plant materials like fruits, spices, herbs and also crude drugs, it has been reported that a cellulose-like signal or a sugar-like spectrum is observable ${ }^{3)-8)}$. The cellulose-like ESR signals are observed in most irradiated plant materials. However, the intensity of signal in some species of plants is low due to the influence of tissue structure and moisture conditions. Meanwhile, in irradiated plant parts with high sugar content, including dried fruit and root, sugar-like ESR spectra were very durable, making it easy to discriminate between irradiated and non-irradiated samples ${ }^{9)}{ }^{10)}$. The observation of sugar-like ESR spectrum can be fully used for detection of irradiated plant materials. However, identification of the specific radicals responsible for individual signals and domination of mono- or disaccharides has not yet been achieved.

In this study, the ESR spectral property of irradiated peony root as a model plant was investigated during development of a system for detection of irradiated medicinal plants containing crystalline sugars. Correlation between radical concentration and sucrose content in the irradiated plant was evaluated.

\section{Materials and methods}

\section{$2 \cdot 1$ Materials}

Peony root (Paeonia lactiflora Pallas) is a popular crude drug in many kampo formulas. The medicinal part is the dried root with peel and small cut pieces. Fracture surface of peony root is dense in texture. Test plant samples were collected from a major cultivation area of the medicinal plant. In this study, seven cultivated peony roots from North Korea and China were obtained from wholesalers. Crystalline mono- and disaccharide reagents : fructose, glu- cose, sucrose were obtained from Nacalai Tesque. The samples were stored at room temperature in the dark $\left(22 \pm 2^{\circ} \mathrm{C}, 50 \pm 10 \% \mathrm{RH}\right)$.

\section{$2 \cdot 2$ Loss on drying test}

Peony root was ground into powder $(<850$ $\mu \mathrm{m})$, and $2 \mathrm{~g}$ of each sample was placed in a weighing bottle and weighed accurately. After drying at $105^{\circ} \mathrm{C}$ for $6 \mathrm{hr}$, the sample was accurately weighed again, and the loss of mass represented the percentage of loss on drying (\%).

\section{$2 \cdot 3$ Analysis of mono- and disaccharides}

Three grams of each sample was added to 15 $\mathrm{mL}$ warm water and sonicated for $5 \mathrm{~min}$. The mixture solution was made up to $25 \mathrm{~mL}$ with $\mathrm{H}_{2} \mathrm{O}$ and centrifuged at $3000 \mathrm{rpm}$ for $5 \mathrm{~min}$. Ethanol was added to bring the $5.0 \mathrm{~mL}$ water layer up to $10 \mathrm{~mL}$. The solution was filtrated through $0.45 \mu \mathrm{m}$ membrane filter. Separation and detection were carried out by the following method ${ }^{11)}$ using a Shimadzu high performance liquid chromatography (HPLC) system (C-VP series) —injection : $6 \mu \mathrm{L}$; column : $\mathrm{NH}_{2} \mathrm{P}-504 \mathrm{E}$ (Asahipak, $4.6 \mathrm{~mm}$ i.d. $250 \mathrm{~mm}$ ), $30^{\circ} \mathrm{C}$; mobile phase : acetonitrile-water (75 : 25) ; flow rate : $1 \mathrm{~mL} / \mathrm{min}$; detection : differential refractometer. Calibration curves were prepared with standard solutions using analytical grade fructose, glucose, sucrose, and each amount of the compound read using the absolute calibration curve method. Measurements were performed two or three times, and the mean values were calculated (relative error, less than $5 \%$ ). Sugar content was calculated from the sum of fructose, glucose and sucrose content.

\section{$2 \cdot 4 \quad$ Irradiation}

Each sample was sealed in polyethylene 

a medicinal plant containing crystalline sugars

bags and irradiated using a dynamitron electron beam accelerator, $5 \mathrm{MeV}$ (Radiation Dynamics, Japan Electron Beam Irradiation Service). EB irradiation was performed in room temperature air (absorbed dose ; $10 \mathrm{kGy}$ ).

\subsection{ESR measurement}

Approximately $0.1 \mathrm{~g}$ of sample was placed in a quartz ESR tube $(5 \mathrm{~mm})$. The tube was then sealed with a plastic paraffin film. ESR measurement was performed using an $\mathrm{X}$-band ESR spectrometer with $100 \mathrm{kHz}$ magnetic field modulator (ES-10, Nikkiso). Measurement was conducted at room temperature under the following conditions:microwave power, $4 \mathrm{~mW}$; sweep width, $15 \mathrm{mT}$; modulation width, 0.2 $\mathrm{mT}$; sweep time, $60 \mathrm{~s}$; time constant, $0.12 \mathrm{~s}$. The g-value was calculated from the signals of the $\mathrm{Mn}^{2+} / \mathrm{MgO}$ marker. ESR intensity was calculated from the area of the double-integrated curve of the observed spectra. Measurements were performed twice, the mean values were calculated (relative error, $1-5 \%$ ) and corrected based on $\mathrm{Mn}^{2+} / \mathrm{MgO}$ signal intensity. The radical concentration was calculated from the area of the double-integrated curve of the ESR spectrum, using 1, 1-diphenyl-2-picrylhydrazyl (in benzene) as the standard.

\section{Results}

\section{$3 \cdot 1$ Water and sugar content}

Water content in dried materials, like crude drugs, changed with humidity in the air. Peony roots are sensitive to humidity and the loss when drying peony root ranged from $8.50 \%$ to 14.0\%. Japanese Pharmacopoeia, an official standard, requires that the loss on drying of peony root is not more than $14.0 \%{ }^{12)}$. Results show that the loss on drying of test samples met the standard. Storage conditions in this
Table 1 Sugar content of peony root

\begin{tabular}{ccc}
\hline sample & $\begin{array}{c}\text { sugar content } \\
(\mathrm{mg} / \mathrm{g} \text { dry })\end{array}$ & \% of \\
\hline a & 243 & 86 \\
$\mathrm{~b}$ & 198 & 68 \\
$\mathrm{c}$ & 152 & 82 \\
$\mathrm{~d}$ & 138 & 66 \\
$\mathrm{e}$ & 88.6 & 64 \\
$\mathrm{f}$ & 67.5 & 53 \\
$\mathrm{~g}$ & 57.3 & 65 \\
\hline Sugar content : sum of sugars & \\
(fructose, glucose and sucrose).
\end{tabular}

study, however, might be slightly higher humidity than that for pharmaceutical use, because a peony root sample near $20 \%$ loss when drying became moldy during storage time (exclusion test sample).

The result of HPLC analysis showed that peony root contained fructose, glucose or sucrose. The sugar content (fructose, glucose and sucrose) ranged from 57.3 to $243 \mathrm{mg} / \mathrm{g}$ dry in peony root. More than $50 \%$ of sugar content in peony root was sucrose (Table 1). Fructose and glucose were found as minor components of sugar in peony root. The sugar content of peony root tended to be higher than that of other medicinal plants (data not shown).

$3 \cdot 2$ ESR property of peony root after irradiation

In non-irradiated peony roots, a weak ESR signal was observed near $\mathrm{g}=2.005$, and the mean value of radical concentration was 8.50 $\pm 3.10\left(\times 10^{15}\right)$ spins/g dry. After $10 \mathrm{kGy}$ irradiation, the ESR line became broader and the intensity increased. A weak cellulose-like signal and a sugar-like spectrum were observed in irradiated peony root, as shown in our previous report $^{6}$.

The change in radical concentration with 

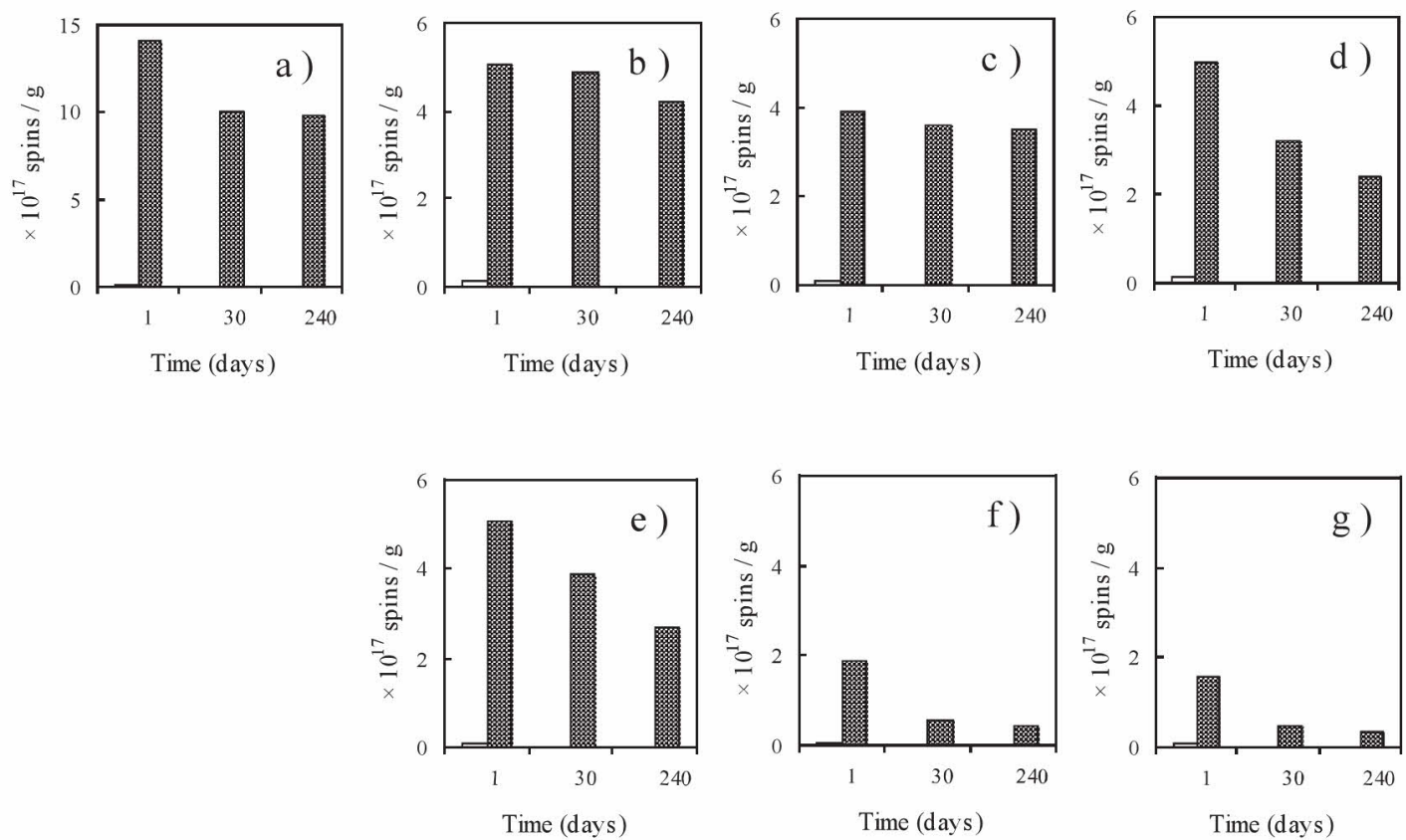

Fig. 1 Change in radical concentration of peony root after $10 \mathrm{kGy}$ EB irradiation. The open bar represents the non-irradiation level.

storage time after irradiation is shown in Fig. 1. Radical concentration in irradiated peony root increased markedly and then decreased for the period up to 30 days post-irradiation; the concentration was stable after that. The radical reduction level at 30 days post-irradiation was ca. $30-90 \%$ of the concentration 1 day post-irradiation. Radical concentration in samples with long storage (240 days post-irradiation) was more than two fold that of non-irradiation level.

Also, few samples changed ESR spectral shape during the period up 30 days post-irradiation. The ESR spectrum of peony root 30 days after $10 \mathrm{kGy}$ irradiation is shown in Fig. 2. Typical sugar-like spectra were observed in all irradiated peony roots on long storage. In $\mathrm{f}$ ) and $\mathrm{g}$ ) in Fig. 2, the signal line shapes were slightly different from that of others : the sugar content was less than $70 \mathrm{mg} / \mathrm{g}$ dry. Moreover, the intensities of spectra in irradiated peony roots were found to significantly correlate to sugar content. The highest ESR intensity was in a), $243 \mathrm{mg} / \mathrm{g}$ dry sugar content; the lowest ESR intensity was in $\mathrm{g}$ ), $57.3 \mathrm{mg} / \mathrm{g}$ dry of sugar content.

\section{$3 \cdot 3$ Regression analysis of ESR intensity af- ter irradiation with content of sucrose}

More than $50 \%$ of sugar content in peony root was sucrose, and the ESR signal line shape of irradiated samples resembled that of irradiated crystalline sucrose (Fig. 3). To show the domination of radical species derived sugars in the ESR spectra of irradiated peony root, regression analysis techniques were applied to sucrose content and ESR intensity of irradiated peony roots. The ESR intensity was calculated using the radical concentration 30 days postirradiation on a stable state.

The linear regression line can describe the 
a)

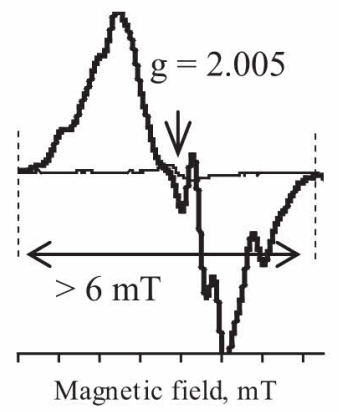

b)

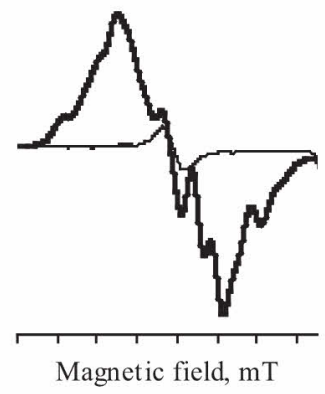

e)

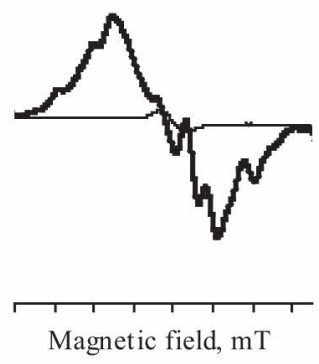

c)

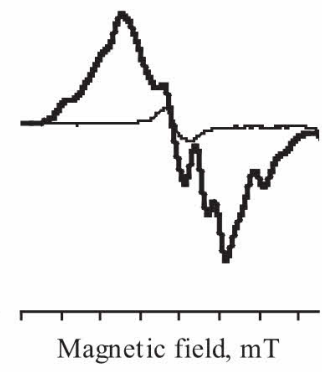

f)

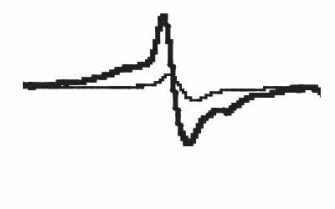

Magnetic field, $\mathrm{mT}$ d)

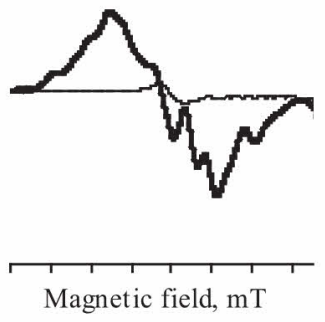

g)

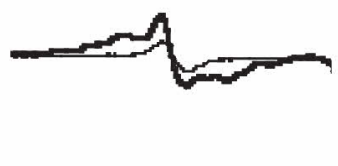

Magnetic field, $\mathrm{mT}$

Fig. 2 ESR spectrum of peony root 30 days after $10 \mathrm{kGy}$ EB irradiation (gain : a, 500 ; b-g, 1600 ).

The bold and solid lines represent the spectra post-irradiation and non-irradiation, respectively.

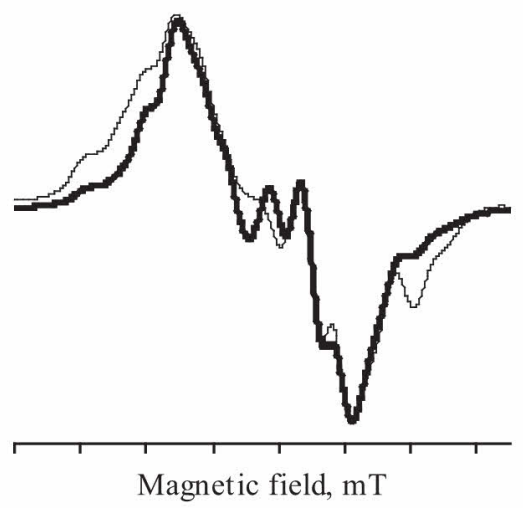

Fig. 3 ESR spectrum of sucrose 30 days after EB irradiation.

The bold line represents the spectrum of irradiated crystalline sucrose. The solid line represents the spectrum of irradiated peony root, Fig. 2 a). increase in the radical concentration of irradiated peony root,

$$
y=a x+b,
$$

where the radical concentration 30 days after $10 \mathrm{kGy} \mathrm{EB}$ irradiation $y$, is expressed as a function of the content of sucrose $x, a$ and $b$ are constants. The content of sucrose $x$ of peony root sample can hence estimate the radical concentration after irradiation. The regression line from least-squares approach of the data obtained for radical concentration from irradiated peony root samples stored for 30 days is given in Fig. 4. The equation representing this line is

$$
\begin{aligned}
& y\left(\times 10^{-17}\right)=0.0645 x-1.91 ; \\
& r=0.906 ; P<0.01 .
\end{aligned}
$$

The line shows a correlation between sucrose 


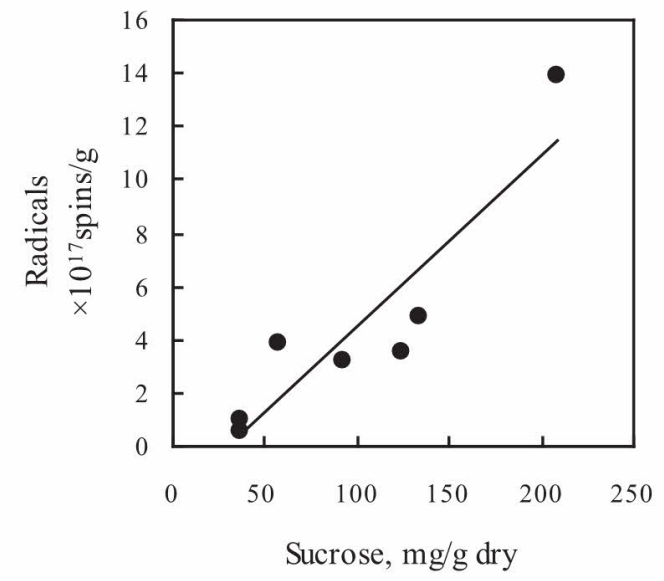

Fig. 4 Correlation between radical concentration 30 days after 10 kGy EB irradiation and sucrose content in peony root. Regression line : $y\left(\times 10^{-17}\right)=0.0645 x-1.91$; correlation coefficient : $0.906 ; P<0.01$.

content and the radical concentration of peony roots 30 days after $10 \mathrm{kGy}$ EB irradiation.

\section{Discussion}

It is important to understand plant constituents and the ESR spectral characteristics of that when analyzing radical species in plant materials post-irradiation. In plant constituents, free radicals trapped in crystalline sugars and fibers are known to remain stable for a very long time ${ }^{13), 14}$. However, environmental parameters such as temperature, humidity and oxygen is significantly assumed to affect the quantity of radical species in samples. Then, storage condition of samples must be maintained as possible.

The sugar content of peony root tended to be higher than that of other medicinal plants. Notably, the high sugar content of peony root (more than $100 \mathrm{mg} / \mathrm{g}$ ) may be attributed to cultivation conditions in addition to plant characteristics. In environmental conditions, temperature and humidity are usually controlled during storage of crude drugs to maintain quality. In the present study, the ESR characterization of peony root after EB irradiation for the reduction of microorganisms (10 kGy) was investigated in a condition similar to storage conditions for pharmaceutical use. As a result, the radical concentration of peony root that increased by irradiation decreased for a period up to 30 days post-irradiation and stabilized after that. Our data show that sugar-like ESR spectra were observed in irradiated peony root on long storage. Therefore, the observation of ESR spectrum of peony root provides strong evidence in support of the irradiation for killing microorganisms.

In particular, more than $50 \%$ of sugar content in peony root was sucrose, and the sugarlike spectra in irradiated peony roots resembled the line of irradiated crystalline sucrose. Our result of regression analysis in Fig. 4 shows that the radical concentration in irradiated peony root closely correlated with sucrose content $(r=0.906)$ and suggests that radicalderived sucrose produced a stable ESR signal in peony root. This finding indicates that the post-irradiation radical concentration correlation based on sucrose content in plant material is predictable. The dose shall be estimated as more than $10 \mathrm{kGy}$ when the observed radical concentration exceeds the predicted radical concentration based on sucrose content in sample on this regression line. In addition, it is reported that the intensity of ESR signals of sucrose showed an increase in proportion to the absorbed dose $^{13)}$. Using the dose response line based on sucrose content, the observed ESR signal intensity of peony root could be converted into an estimate of absorbed dose. Besides, such a calibration model using sugar content in the sample is expected to be utilized 
with other plant materials observable stable sugar-like ESR spectrum, including fruit. For that purpose, it is necessary to identify the radical component derived from crystalline sugar in irradiated plant material and to determine a relationship between the ESR intensity, sugar content and the given dose.

\section{Conclusions}

In irradiated peony roots $(10 \mathrm{kGy})$, the ESR line near $\mathrm{g}=2.005$ became broader, and sucrose-like spectra were observable on long storage. In peony root, the radical concentration after 30 days irradiation correlated with sucrose content. This result suggests that radical-derived sucrose produced a stable ESR signal and the radical concentration correlation to sucrose content in irradiated plant material is predictable. ESR detection of peony root containing sugar provides strong evidence in support of irradiation in order to kill microorganisms.

\section{References}

1) EN 1787, Foodstuffs, Detection of Irradiated Food Containing Cellulose, Method by ESR Spectroscopy, European Committee for Standardization, Belgium (2000)

2) EN 13708, Foodstuffs, Detection of Irradiated Food Containing Crystalline Sugar by ESR Spectroscopy, European Committee for Standardization, Belgium (2001)

3) Tabner, B. and Tabner, V. A., Stable radicals observed in the flesh of irradiated citrus fruits by electron spin resonance spectroscopy for the first time, Radiat. Phys. Chem., 47, 601-605(1996)

4) Raffi, J., Yordanov, N. D., Chabane, S., Douifi, L., Gancheva, V. and Ivanova, S., Identification of irradiation treatment of aromatic herbs, spices and fruits by electron paramagnetic resonance and thermoluminescence, Spectrochimica Acta Part A, 56, 409-416 (2000)

5) Korkmaz, M. and Polat, M., Radical kinetics and characterization of the free radicals gamma irradiated red pepper, Radiat. Phys. Chem., 62, 411421 (2001)

6) Yamaoki, R., Kimura, S., Aoki, K. and Nishimoto, $\mathrm{S}$., Detection of electron beam irradiated crude drugs by electron spin resonance (ESR), RADIOISOTOPES, 56, 163-172 (2007)

7) Kikuchi, M., Hussain, M. S., Morishita, N., Ukai, M., Shimoyama, Y. and Kobayashi, Y., An ESR study of radicals induced in irradiated fresh mango, RADIOISOTOPES, 58, 789-797 (2009)

8) Yoshida, T., Hironiwa, T., Masumizu, T., Hara, H., Okano, M. and Miyahara, M., Study of detection of irradiated dried fruits by ESR spectroscopy, RADIOISOTOPES, 59, 463-469(2010)

9) Esteves, M. P., Andrade, M. E. and Empis, J., Detection of prior irradiation of dried fruits by electron spin resonance(ESR), Radiat. Phys. Chem., 55, 737-742 (1999)

10) Yordanov, N. D. and Pachova, Z., Gamma-irradiated dry fruits : An example of a wide variety of long-time dependent EPR spectra, Spectrochimica Acta, Part A, 63, 891-895 (2006)

11) Yasui, T., Toda, C., Nagano, H. and Ishihara, H., Determination of oligosaccharides in foods for specified health use by HPLC, Shokuhin Eiseigaku Zasshi, 37, 29-37(1996)

12) The Japanese Pharmacopoeia Fifteenth Edition, Official Monographs, Crude Drugs, p.1295, p.1328, Ministry of Health, Labour and Welfare, Tokyo, Japan (2006)

13) Nakajima, T., Sugar as an emergency populance dosimeter for radiation accidents, Health Physics, 55, 951-955 (1988)

14) Yordanov, N. D. and Georgieva, E., EPR and UV spectral study of gamma-irradiated white and burned sugar, fructose and glucose, Spectrochimica Acta, Part A, 60, 1307-1314 (2004) 